\title{
émulations
}

\section{François-Ronan Dubois - Introduction aux Porn Studies}

\author{
Anne-Charlotte Millepied
}

Émulations - Revue de sciences sociales

2019, Comptes rendus critiques, En ligne.

\section{Article disponible à l'adresse suivante}

https://ojs.uclouvain.be/index.php/emulations/article/view/crmillepied

\section{Pour citer cet article}

Anne-Charlotte Millepied, « François-Ronan Dubois - Introduction aux Porn Studies », Émulations, en ligne. Mise en ligne le 13 juin 2019.

DOI : 10.14428/emulations.cr.065

Distribution électronique : Université catholique de Louvain (Belgique) : ojs.uclouvain.be

(C) Cet article est mis à disposition selon les termes de la Licence Creative Commons Attribution, Pas d'Utilisation Commerciale 4.0 International. http://creativecommons.org/licenses/by-nc/4.0/

Éditeur : Émulations - Revue de sciences sociales / Presses universitaires de Louvain https://ojs.uclouvain.be/index.php/emulations

ISSN électronique : 1784-5734

PUL PRESSES 


\title{
François-Ronan Dubois - Introduction aux Porn Studies
}

\begin{abstract}
Anne-Charlotte Millepied ${ }^{1}$
Recensé : François-Ronan Dubois, Introduction aux Porn Studies, Bruxelles, Les Impressions Nouvelles, 2014,128 p.

Dans cet ouvrage concis, François-Ronan Dubois, agrégé de lettres modernes et docteur en littérature française, propose une introduction aux études sur la pornographie. Il faut d'emblée souligner son ouverture à un lectorat non académique : son propos est clair et accessible. L'objectif de l'auteur est de présenter de manière simplifiée les principaux enjeux de débat autour de la pornographie, objet polémique tant dans l'espace public que dans le champ académique. La nécessité d'un discours savant sur la pornographie se fait sentir face à l'expression des fantasmes, des idées reçues et des "paniques morales» (Bozon, 2012) que la pornographie tend à soulever. L'auteur cherche à présenter ces débats de façon complète et cohérente, sans pour autant se cacher derrière une posture objective et neutre. Cependant, bien qu'il se « situe plutôt dans [le camp] des porn studies » (p. 56), il cherche dans cet ouvrage à se positionner à l'extérieur des débats qu'il présente, et, on y reviendra en conclusion, il donne plus de place aux enjeux juridiques et éthiques de la représentation pornographique qu'à ses enjeux socio-culturels.

Le premier chapitre est consacré à la description des différentes formes que prend la pornographie, un objet aux frontières floues, tant dans les débats publics que chez les experts ou dans les textes de loi. Dubois adopte d'abord une perspective historique et souligne la difficulté de donner un acte de naissance à la pornographie. Il propose un survol historique de l'Antiquité à nos jours, qui permet de mettre en lumière le caractère socialement et culturellement situé des définitions de la pornographie et de ses frontières. Parmi les formes de la pornographie, Dubois distingue le texte, l'image fixe, l'image mouvante et internet. Il affiche le souci de présenter un large panorama de ce qui existe en pornographie, ce qui lui permet de ne pas considérer cette dernière comme un bloc monolithique et ainsi de dépasser l'horizon de la pornographie hétérosexuelle dominante, sur-représentée dans la plupart des débats publics. Le champ pornographique est en effet extrêmement divers et traversé par des tensions internes. L'auteur ne manque ainsi pas de mentionner les contre-discours qui
\end{abstract}

${ }^{1}$ Doctorante en sociologie à l'École des Hautes Études en Sciences Sociales (EHESS), au sein de I'Institut de recherche interdisciplinaire sur les enjeux sociaux (Iris), et à l'Université de Genève (UNIGE), au sein de l'Institut des Études Genre. 
s'opposent au discours de dénonciation de l'antipornographisme et les alternatives gaies, lesbiennes et queer, peu visibilisées, qui les accompagnent. Il s'attarde sur le support filmique et sur internet, qui représente une nouvelle étape dans l'histoire de la pornographie caractérisée par une abondance du matériau et par la visibilité donnée à des objets d'ordinaire exclus de la diffusion traditionnelle de la pornographie (par exemple la production des amateurs).

Le deuxième chapitre rend compte des études savantes sur la pornographie et les polémiques au sein du monde académique. Les porn studies, que l'on peut littéralement traduire "études pornographiques", se distinguent parce qu'elles "s'opposent à d'autres perspectives savantes sur la question» (p.55). Pour contextualiser l'agencement des différentes approches savantes de la pornographie, l'auteur présente le contexte américain des années 1970-1980, qui a fortement influencé le cadre des débats académiques ultérieurs sur la pornographie. Face à l'alliance de circonstance entre la droite chrétienne et une partie du féminisme radical, une position féministe dite « pro-sexe » s'est constituée pour mettre en avant la possibilité d'imaginer et créer une pornographie non oppressive et subversive. Cette période des "guerres du sexe » $(\text { sex wars })^{2}$ a donné lieu à une légitimation, essentiellement à l'université, des positions pro-pornographiques, tandis que le discours conservateur a perdu de son influence bien qu'il reste très actif dans la sphère médiatique et publique.

Ce contexte posé, Dubois choisit ensuite de distinguer, au sein des études savantes sur la pornographie, celles relevant du domaine juridique, celles se rapportant à la psychopathologie, et celles appartenant aux études cinématographiques. La perspective juridique est importante car elle définit le cadre légal qui permet d'établir ou non une censure. Elle s'appuie sur les enquêtes psychopathologiques car l'élaboration du cadre juridique nécessite des expertises, et il s'avère que les experts en psychopathologie sont principalement mobilisés. Le plus souvent convoqués pour asseoir les positions antipornographiques, il s'agit pour ces experts de prouver que la pornographie joue un rôle sur les comportements sexuels déviants et violents. Les études cinématographiques, quant à elles, se caractérisent globalement par une approche pro-pornographique, qui se présente aussi comme un alter-pornographisme. C’est au sein de ce champ d'études que se développent les premières critiques du féminisme antipornographie alors dominant (au sein du mouvement féministe) et que s'élabore une position féministe ouverte aux sexualités et expressions de genre minoritaires ${ }^{3}$. L'ouvrage de référence de Linda Williams (1989) a contribué, selon Dubois, à donner une légitimité académique à la position pro-sexe, et représente selon l'auteur un tournant idéologique tout autant qu'un renouveau méthodologique. Elle a ouvert la voie à une nouvelle génération d'études qui se caractérisent par une approche

\footnotetext{
${ }^{2}$ Ces « guerres du sexe » se sont avant tout focalisées sur la pornographie et la prostitution.

${ }^{3}$ Au-delà de la pornographie, les études cinématographiques sont à l'origine de l'expression «queer theory », proposée par Teresa de Lauretis (2007).
} 
plutôt culturaliste, une forte interdisciplinarité et un attachement à une posture critique.

Le troisième et dernier chapitre, qui porte sur les conséquences d'un savoir pornographique, questionne la dimension éthique soulevée par la pornographie. Pour replacer les études pornographiques dans une démarche plus large d'historicisation de la sexualité, il se livre à une présentation rapide de la pensée dite " poststructuraliste », " déconstructionniste », « queer » ou « postmoderne » autour des écrits des philosophes Friedrich Nietzsche, Michel Foucault et Judith Butler. Ce détour un peu long rompt avec le reste de l'ouvrage qui prenait soin, jusque-là, d'éviter les références théoriques, mais permet à l'auteur de montrer que les études pornographiques sont héritières d'une école philosophique qui s'attache à penser les conditions de la morale et de la sexualité. Il souligne ainsi l'« enjeu éthique, moral et politique majeur au sein des études pornographiques $»$ (p. 95).

Dans la conclusion, l'auteur soulève un élément qui nous semble primordial mais qui ne bénéficie néanmoins pas d'un développement: « la question est parfois moins celle de la pornographie que celle de la culture de l'élite et de la manière dont l'élite traite la culture plus populaire » (p. 109). En effet, les débats actuels autour de la pornographie se jouent aussi sur le terrain de la légitimité culturelle ; ils sont révélateurs des luttes autour de la légitimation de la représentation pornographique comme catégorie artistique à part entière. On comprend alors l'intérêt des recherches sociologiques qui placent au cœur de leurs analyses les rapports de classe tout en adoptant une perspective intersectionnelle. Celle-ci permet de comprendre l'imbrication des différents rapports sociaux (classe, genre, race, âge, entre autres) qui se jouent dans la production, la réception et le traitement social de la pornographie. Les enquêtes critiques en sciences sociales sont ainsi nécessaires pour mettre au jour les mécanismes à l'œuvre dans la pornographie concernant la reproduction des rapports de domination ou bien leur déstabilisation et leur subversion. En France, de telles enquêtes se sont d'ailleurs multipliées ces dernières années.

De façon générale, cet ouvrage est certes une introduction aux principales lignes de débat concernant la pornographie dans l'espace public et dans l'espace académique, mais il ne constitue pas véritablement une « introduction aux Porn Studies». Une telle démarche aurait supposé de donner un meilleur aperçu de la littérature académique en mentionnant les travaux " classiques » aussi bien que des recherches plus récentes. Bien que l'auteur précise à la fin de l'avant-propos que son «ambition n'a rien d'universitaire » (p.16), on regrette toutefois l'absence de références bibliographiques pour soutenir le texte, que ce soit concernant les études cinématographiques (à l'exception de Linda Williams) ou les approches juridiques et psychopathologiques,

${ }^{4}$ On peut par exemple faire référence aux travaux de Béatrice Damian-Gaillard (2012); Mathieu Trachman (2012) ou de Florian Vörös (2015). En linguistique et études du discours, on peut se référer au numéro "La pornographie et ses discours » de la revue Questions de communication (2014) ainsi qu'au travail de Marie-Anne Paveau (2014). 
dont la présentation ne s'appuie sur aucun exemple précis. Dans la même logique, la bibliographie indicative présente en fin d'ouvrage aurait mérité d'être plus fournie et plus représentative du champ étudié. On pense par exemple aux travaux de Feona Attwood (2005 ; 2011), Carolyn Bronstein (2011), Jane Juffer (1998), Walter Kendrick (1987), Laura Kipnis (1998), Susanna Paasonen (2011), Annie Stora-Lamarre (1989), ou encore à plusieurs ouvrages collectifs qui traitent de la pornographie au prisme du genre (entre autres : Church-Gibson, Gibson (dir.) 1993; Segal, McIntosh (dir.), 1993; Taormino, Penley, Parreñas Shimizu, Miller-Young (dir.), 2013)5.

Malgré cette lacune, l'ouvrage de François-Ronan Dubois fournit tout de même une bonne introduction aux différents enjeux sociaux, politiques, juridiques et éthiques de l'objet " pornographie ». Sa posture dépassionnée est particulièrement bienvenue dans le cadre d'une présentation qui se veut accessible au lectorat non académique ou peu familier à la question.

\section{Bibliographie}

ATTwOOD F. (2005), « What do people do with porn? Qualitative research into the consumption, use, and experience of pornography and other sexually explicit media ", Sexuality and Culture, vol. 9, n 2, p. 65-86.

AtTwood F. (2011), « The Paradigm Shift: Pornography Research, Sexualization and Extreme Images », Sociology Compass, vol. 5, n 1, p. 13-22.

Bozon M. (2012), « Autonomie sexuelle des jeunes et panique morale des adultes. Le garçon sans frein et la fille responsable », Agora débats/jeunesses, vol. 1, nº 60, p. 121134.

Bronstein C. (2011), Battling Pornography: The American Feminist Anti-Pornography Movement, 1976-1986, Cambridge, Cambridge University Press.

Church-Gibson P., Gibson R. (dir.) (1993), Dirty Looks: Women, Pornography, Power, Londres, British Film Institute.

Damian-Gaillard B. (2012), "Entretiens avec des producteurs de la presse pornographique: des rencontres semées d'embûches... », Sur le journalisme, vol.1, $\mathrm{n}^{\circ} 1$, p. 84-95.

De LAURETIS T. (2007), Théorie queer et cultures populaires. De Foucault à Cronemberg, Paris, La Dispute.

${ }^{5}$ Pour plus d'exemples, voir la notice «Pornographie » de l'Encyclopédie critique du genre, rédigée par Mathieu Trachman et Florian Vörös (2016); voir aussi l'anthologie dirigée par Florian Vörös (2015) et son excellente introduction (2015: 5-31). 
JufFer J. (1998), At Home with Pornography: Women, Sex and Everyday Life, New York, New York University Press.

KENDRICK W. (1996 [1987]), The Secret Museum: Pornography in Modern Culture, Berkeley, University of California Press.

KIPNIS L. (2007 [1996]), Bound and Gagged: Pornography and the Politics of Fantasy in America, Durham, Duke University Press.

PAasonen S. (2011), Carnal Resonance: Affect and Online Pornography, Cambridge, MIT Press.

Paveau M-A. (2014), Le discours pornographique, Paris, La Musardine.

Paveau M-A, Perea F. (dir.) (2014), «La pornographie et ses discours », Questions de communication, $\mathrm{n}^{\circ} 26$.

Segal L., McIntosh M. (dir.) (1993), Sex Exposed: Sexuality and the Pornography Debate, New Brunswick, Rutgers University Press.

StORA-LAMARre A. (1989), L'Enfer de la IIIe République : censeurs et pornographes (1881-1914), Paris, Imago.

Taormino T., Penley C., Parreñas Shimizu C., Miller-Young M. (dir.) (2013), The Feminist Porn Book: The Politics of Producing Pleasure, New York, The Feminist Press.

TRAChMAN M., VÖRÖS F. (2016), « Pornographie », in J. Rennes (dir.), Encyclopédie critique du genre. Corps, sexualité, rapports sociaux, Paris, La Découverte, p. 479-487.

TRAChman M. (2012), Le travail pornographique. Enquête sur la production de fantasmes, Paris, La Découverte.

VÖRÖs F. (2014), Les usages sociaux de la pornographie en ligne et les constructions de la masculinité. Une sociologie matérialiste de la réception des médias, thèse de doctorat en sociologie, Paris, École des Hautes Études en Sciences Sociales.

VöRös F. (dir.) (2015), Cultures pornographiques. Anthologie des Porn Studies, Paris, Éditions Amsterdam.

VöRÖS F. (2015), « Introduction. Le porno à bras le corps. Genèse et épistémologie des porn studies », in F. VöRÖs (dir.), Cultures pornographiques. Anthologie des Porn Studies, Paris, Éditions Amsterdam, p. 5-31.

Williams L. (1999 [1989]), Hard Core: Power, Pleasure and the Frenzy of the Visible, Berkeley, Los Angeles/Londres, University of California Press. 